\title{
Estimation of Road Inclination and Bank Angle in Automotive Vehicles
}

\author{
Håvard Fjær Grip ${ }^{\star} \quad$ Lars Imsland ${ }^{\dagger} \quad$ Tor A. Johansen ${ }^{\star} \quad$ Jens C. Kalkkuhl ${ }^{\ddagger} \quad$ Avshalom Suissa ${ }^{\ddagger}$ \\ ^Department of Engineering Cybernetics, Norwegian University of Science and Technology, 7491 Trondheim, Norway \\ ${ }^{\dagger}$ Cybernetica, 7038 Trondheim, Norway \\ $\ddagger$ Daimler Group Research and Advanced Engineering, 71059 Sindelfingen, Germany
}

\begin{abstract}
We extend an observer design for estimation of the vehicle sideslip angle on horizontal surfaces to include estimation of the road inclination angle and the road bank angle. The design makes use of a nonlinear road-tire friction model, and the nonlinearity of the road-tire friction forces are taken into account in the theoretical analysis of the design. Using an absolute-stability argument we show that, under a set of technical assumptions, the origin of the observer error dynamics is globally exponentially stable. Taking unknown road-surface conditions into account, we discuss simultaneous estimation of the road bank angle and a road-tire friction parameter, which is complicated by the dependence of the friction-parameter estimation on lateral excitation of the vehicle. To improve performance on low-friction surfaces, we modulate the observer gains based on a set of practical conditions. Using experimental data from a passenger car, we investigate the performance of the approach.
\end{abstract}

\section{INTRODUCTION}

For implementation of automotive control algorithms, accurate information about the state of the vehicle and its surroundings is important. Whereas some quantities can be obtained by direct measurement, others are difficult to measure because of high cost or impracticality. Among the quantities that are not measured in production vehicles is the angle between the heading of the vehicle and the direction of travel, known as the vehicle sideslip angle. To estimate the vehicle sideslip angle, it is common to estimate the longitudinal and lateral velocities of the vehicle, denoted $v_{x}$ and $v_{y}$, and to calculate the sideslip angle as $\arctan \left(v_{y} / v_{x}\right)$.

The longitudinal and lateral velocities are influenced by gravity components due to slanting of the road surface. For velocity observers that rely on the vehicle's dynamic equations of motion, knowledge about the slanting of the road surface is therefore important. Nevertheless, sideslip estimation designs often assume that the road surface is flat and horizontal. Designs that take the slanting of the road surface into account include [1]-[3].

Knowledge about the slanting of the road surface is of interest for purposes other than sideslip estimation, and several designs focus specifically on estimation of the road bank angle. These include [4], which is based on transfer functions from the steering angle and road bank angle to the yaw rate and lateral acceleration, and [5], [6], which use an extended Kalman filter (EKF) to estimate the sideslip

This research is supported by the European Commission STREP project Complex Embedded Automotive Control Systems, contract 004175, and the Research Council of Norway. angle, which is in turn used in a linear unknown-input observer to estimate the road bank angle. Several GPS-based designs, such as [7], [8], estimate the road inclination or bank angle. In this paper, we do not assume availability of GPS measurements.

\section{A. Contributions of This Paper}

Sideslip estimation designs found in literature are usually based on linear or quasi-linear techniques. Most commonly, an EKF is employed. Although often effective, the EKF is difficult to analyze because the algorithm is derived through linearization of the prediction error [9]. Other potential disadvantages associated with the EKF are high computational complexity - due to the need to solve the Riccati equation at each time step — and a large number of tuning parameters.

In [10], [11], the authors presented a nonlinear vehicle sideslip observer for a vehicle traveling on a flat, horizontal surface. The main features of this observer are a reduction in computational complexity and in the number of tuning parameters compared to an EKF. In addition, analysis of stability and robustness in [10], [11] is carried out in a nonlinear setting. Inspired by the theory of nonlinear unknown-input observers, an extension of [10] for estimating the bank angle is developed in [12]; however, the nature of the disturbance introduced by road surface slanting suggests that something similar to regular integral action is appropriate. In this paper, we present such a solution, extending the design from [10], [11] to include estimation of the road inclination and bank angles. We analyze the stability of the design, taking the nonlinearity of the road-tire friction forces into account.

\section{B. Sensor Configuration}

In our design, we assume that measurements of the longitudinal and lateral accelerations, wheel speeds, steering wheel angle, and yaw rate are available. These measurements can be considered standard in modern passenger cars with an electronic stability control system (ESC).

\section{ROAD-TIRE FRICTION}

When a car turns, the tires become misaligned with the direction of travel. The angle between the direction the tire is pointing in and the velocity vector of the vehicle above the center of the tire is called the tire slip angle. Fig. 1 illustrates the tire slip angle $\alpha_{1}$ for the front-left wheel. A nonzero tire slip angle gives rise to friction forces in the lateral direction of the tire, illustrated by $F_{y, 1}$ in Fig. 1 . The longitudinal 


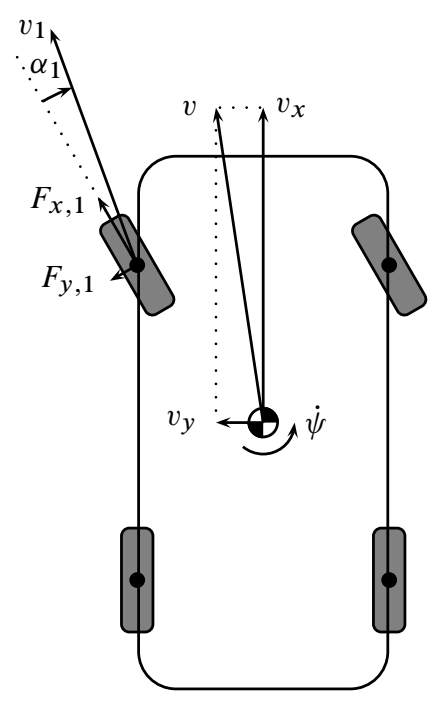

Fig. 1. Illustration of the car

tire slip, defined as a normalized difference between the circumferential velocity of the tire and the velocity of the tire center, gives rise to longitudinal friction forces, illustrated by $F_{x, 1}$ in Fig. 1. Collectively, we refer to the longitudinal tire slip and lateral tire slip angle as the tire slips.

During normal driving the road-tire friction forces are approximately linear with respect to the tire slips. When the tire slips become large, however, the road-tire friction forces saturate, meaning that an increase in the tire slips does not result in a corresponding increase in friction forces.

A key component of the design presented in this paper is a road-tire friction model. The road-tire friction model takes measurements and observer estimates as inputs, and returns estimates of the road-tire friction forces. To account for the nonlinearity of the road-tire friction forces for large tire slips, a fully nonlinear model is needed. A widely used example of a nonlinear tire model is the magic formula [13].

The observer presented in this paper is not designed with a particular road-tire friction model in mind. Instead, we assume that a nonlinear friction model satisfying some physically reasonable properties is used, and we base the design and analysis on these properties. The experiments are carried out using a proprietary road-tire friction model of a complexity comparable to the magic formula.

\section{A. Monotonicity}

According to Newton's second law, the vehicle's acceleration in a particular direction is equal to the sum of the forces acting in that direction, divided by the mass. For a vehicle traveling on a horizontal surface, the most significant forces acting in the road-surface plane are the road-tire friction forces, and we ignore smaller influences such as wind forces and air resistance. The road-tire friction forces are functions of the tire slips, which in turn are functions of the vehicle velocities. Consequently, the measurements of the vehicle accelerations are indirect measurements of the vehicle velocities.
To see how the connection between the accelerations and the velocities can be exploited, we consider what happens if we change the lateral velocity $v_{y}$ while keeping everything else constant. When the lateral velocity increases, it causes the tire slip angles to decrease. At each wheel, the decrease in the tire slip angle generally leads to a decrease in the lateral road-tire friction force. As a net result, the acceleration $a_{y}$ decreases when the lateral velocity $v_{y}$ increases, meaning that the relationship between $a_{y}$ and $v_{y}$ can be modeled as monotonic. ${ }^{1}$ It is demonstrated in [10] that, except in some particular cases, the partial derivative $\partial a_{y} / \partial v_{y}$ is smaller than some negative number. In [10], the monotonicity property is exploited to make $a_{y}$ an indirect measurement of $v_{y}$.

When the vehicle is moving on a slanted surface, gravity components influence the velocity of the vehicle, in addition to the road-tire friction forces. Nevertheless, because the accelerometers are influenced by the same gravity forces as the vehicle itself, the measured accelerations still correspond to the sum of the road-tire friction forces divided by the mass. This means that, even on a slanted surface, we can still exploit the monotonicity property of the measured lateral acceleration as an indirect measurement of $v_{y}$.

\section{Velocity Estimation on Horizontal Surface}

Before introducing the design for estimation of the inclination and bank angles, we briefly review the design from [10]. For a vehicle traveling on a horizontal surface, the vehicle velocities are governed by the equations of motion [14]

$$
\begin{aligned}
& \dot{v}_{x}=a_{x}+\dot{\psi} v_{y}, \\
& \dot{v}_{y}=a_{y}-\dot{\psi} v_{x},
\end{aligned}
$$

where $v_{x}$ and $v_{y}$ are the longitudinal and lateral velocities, $a_{x}$ and $a_{y}$ are the longitudinal and lateral accelerations, and $\dot{\psi}$ is the yaw rate. Throughout the rest of this paper, a hat indicates an estimated quantity, and a tilde indicates an estimation error, meaning that $\hat{v}_{x}$ is an estimate of $v_{x}$, and $\tilde{v}_{x}:=v_{x}-$ $\hat{v}_{x}$. We discuss the longitudinal-velocity estimate $\hat{v}_{x}$ and the lateral-velocity estimate $\hat{v}_{y}$ separately, ignoring at first the interconnections between the two estimates.

\section{A. Longitudinal-Velocity Estimation}

The longitudinal-velocity estimate is given by

$$
\dot{\hat{v}}_{x}=a_{x}+\dot{\psi} \hat{v}_{y}+K_{v_{x}}(t)\left(v_{x, \text { ref }}-\hat{v}_{x}\right),
$$

where $K_{v_{x}}(t) \geq K_{v_{x}, \text { min }}>0$ is a time-varying gain, and $v_{x \text {,ref }}$ is a reference velocity calculated from the wheel speeds.

To calculate the reference velocity, we use the four wheel speeds, the steering wheel angle, and the yaw rate to obtain four separate estimates of the longitudinal velocity. The reference velocity is calculated as a weighted average of these four velocity estimates, and the gain $K_{v_{x}}(t)$ is made to reflect the quality of the reference velocity. When the quality of the reference velocity is deemed to be low, the

\footnotetext{
${ }^{1}$ See Section VI for further remarks on this topic.
} 
gain is reduced, making the observer less reliant on the reference velocity, and more reliant on integration of the system equations. The primary factor in determining the quality of the reference velocity is the spread in the four velocity estimates from the wheel speeds.

Ignoring the lateral-velocity error $\tilde{v}_{y}$, global exponential stability of the longitudinal-velocity error is easily proven when we assume that $v_{x \text {,ref }}$ represents the true longitudinal velocity.

\section{B. Lateral-Velocity Estimation}

For the lateral velocity, we start by introducing $\hat{a}_{y}\left(t, \hat{v}_{x}, \hat{v}_{y}\right)$, which denotes an estimate of the lateral acceleration $a_{y}$. The estimate $\hat{a}_{y}\left(t, \hat{v}_{x}, \hat{v}_{y}\right)$ is formed by using the nonlinear friction model for each wheel, where the measurements of the steering wheel angle, yaw rate, and wheel speeds, as well as the estimated velocities $\hat{v}_{x}$ and $\hat{v}_{y}$, are used as inputs. The friction forces modeled for each wheel are added up in the lateral direction of the vehicle and divided by the mass, resulting in the lateral-acceleration estimate $\hat{a}_{y}\left(t, \hat{v}_{x}, \hat{v}_{y}\right)$. The $t$ in $\hat{a}_{y}\left(t, \hat{v}_{x}, \hat{v}_{y}\right)$ denotes the dependence of $\hat{a}_{y}$ on time-varying signals such as the steering wheel angle. We also write $\tilde{a}_{y}\left(t, \tilde{v}_{x}, \tilde{v}_{y}\right):=a_{y}-\hat{a}_{y}\left(t, \hat{v}_{x}, \hat{v}_{y}\right)$.

The lateral-velocity estimate is given by

$$
\dot{\hat{v}}_{y}=a_{y}-\dot{\psi} \hat{v}_{x}-K_{v_{y}}\left(a_{y}-\hat{a}_{y}\left(t, \hat{v}_{x}, \hat{v}_{y}\right)\right),
$$

where $K_{v_{y}}$ is a positive gain. Analysis of (3) is based on the following assumption:

Assumption 1: There exists a continuous function $\delta\left(t, \tilde{v}_{y}\right) \geq \delta_{\min }>0$ such that, if $\tilde{v}_{x}=0$, then

$$
\tilde{a}_{y}\left(t, 0, \tilde{v}_{y}\right)=-\delta\left(t, \tilde{v}_{y}\right) \tilde{v}_{y} .
$$

Assumption 1 is justified by the previous discussion of the monotonic relationship between $a_{y}$ and $v_{y}$, by using the mean value theorem. Using Assumption 1, global exponential stability of the lateral-velocity error is easily verified when the longitudinal-velocity error $\tilde{v}_{x}$ is ignored.

\section{Combined Longitudinal- and Lateral-Velocity Estimation}

When we take the interconnections between the two observer equations (2), (3) into account, we require an additional assumption:

Assumption 2: There exists a continuous, uniformly bounded function $\kappa\left(t, \tilde{v}_{x}, \tilde{v}_{y}\right)$ such that

$$
\hat{a}_{y}\left(t, v_{x}, \hat{v}_{y}\right)-\hat{a}_{y}\left(t, \hat{v}_{x}, \hat{v}_{y}\right)=\kappa\left(t, \tilde{v}_{x}, \tilde{v}_{y}\right) \tilde{v}_{x} .
$$

Using Assumption 2, we can verify that the origin of the error dynamics for the observer (2), (3) is globally exponentially stable, provided $K_{v_{x} \text {, min }}$ is chosen large enough to dominate the cross-terms that occur because of the interconnections. The observer is also input-to-state stable with respect to

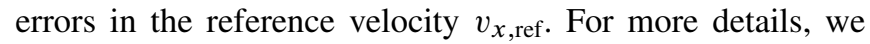
refer to [10].

\section{INCLINATION AND BANK ANGLE}

We now consider the presence of nonzero inclination and bank angles. To define these angles precisely, we say that the orientation of the road surface is obtained from the horizontal position by a rotation around the vehicle's $y$-axis equal to the inclination angle $\Theta$, and a subsequent rotation around the $x$-axis equal to the bank angle $\Phi$. The inclination and bank angles cause gravity components to appear in the equations of motion, and we rewrite (1) as [3]

$$
\begin{aligned}
& \dot{v}_{x}=a_{x}+\dot{\psi} v_{y}+g \sin (\Theta), \\
& \dot{v}_{y}=a_{y}-\dot{\psi} v_{x}-g \cos (\Theta) \sin (\Phi),
\end{aligned}
$$

where $g$ is the acceleration of gravity. It is important to note that in (5), $a_{x}$ and $a_{y}$ denote the accelerations measured by the accelerometers. We assume that the inclination and bank angles vary slowly enough compared to the dynamics of the system to be modeled as constants.

\section{A. Inclination Angle}

We define $\theta_{\mathrm{i}}=\sin (\Theta)$, and introduce an estimate $\hat{\theta}_{\mathrm{i}}$ of $\theta_{\mathrm{i}}$. Although we can solve for the inclination angle by writing $\Theta=\arcsin \left(\theta_{\mathrm{i}}\right)$, typical inclination angles are small enough that $\Theta \approx \theta_{\mathrm{i}}$. The estimate $\hat{\theta}_{\mathrm{i}}$ is therefore considered an estimate of the inclination angle. We use $\hat{\theta}_{\mathrm{i}}$ to compensate for the disturbance, by letting

$$
\dot{\hat{v}}_{x}=a_{x}+\dot{\psi} \hat{v}_{y}+g \hat{\theta}_{\mathrm{i}}+K_{v_{x}}(t)\left(v_{x, \mathrm{ref}}-\hat{v}_{x}\right),
$$

where $\hat{\theta}_{\mathrm{i}}$ is given by

$$
\dot{\hat{\theta}}_{\mathrm{i}}=K_{\theta_{\mathrm{i}}} K_{v_{x}}(t)\left(v_{x, \mathrm{ref}}-\hat{v}_{x}\right),
$$

with $K_{\theta_{\mathrm{i}}}$ a positive gain. If the gain in (7) were constant, the inclination-angle estimation would be recognized as regular integral action. Instead, we let the gain in (7) vary in direct proportion to $K_{v_{x}}(t)$. The reason for using a time-varying gain is the same as in the longitudinal-velocity estimation itself, namely that we wish to rely less on the reference velocity $v_{x \text {,ref }}$ whenever it is of poor quality.

To justify this approach, we use the theory of absolute stability [15, Ch. 7.1]. We split the gain $K_{v_{x}}(t)$ into a constant and a time-varying part by writing $K_{v_{x}}(t)=a+$

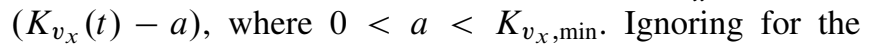
moment the lateral-velocity error $\tilde{v}_{y}$, and assuming that $v_{x \text {,ref }}$ represents the true longitudinal velocity, we write the error dynamics of the longitudinal-velocity and inclination-angle estimates as the interconnection of the linear time-invariant (LTI) system

$$
\begin{gathered}
\dot{\tilde{v}}_{x}=-a \tilde{v}_{x}+g \tilde{\theta}_{\mathrm{i}}+u, \\
\dot{\tilde{\theta}}_{\mathrm{i}}=-K_{\theta_{\mathrm{i}}} a \tilde{v}_{x}+K_{\theta_{\mathrm{i}}} u
\end{gathered}
$$

with the time-varying sector function $u=-\left(K_{v_{x}}(t)-a\right) \tilde{v}_{x}$.

Lemma 1: If the gain $K_{\theta_{\mathrm{i}}}$ is chosen such that $0<K_{\theta_{\mathrm{i}}}<$ $a / g$, then the origin of (8) is globally exponentially stable.

Proof: The function $\phi_{x}\left(t, \tilde{v}_{x}\right)=\left(K_{v_{x}}(t)-a\right) \tilde{v}_{x}$ belongs to the sector $[0, \infty]$, because $\phi_{x}\left(t, \tilde{v}_{x}\right) \tilde{v}_{x} \geq 0[15$, Def. 6.2]. The transfer function from $u$ to $\tilde{v}_{x}$ is given by

$$
G(s)=\frac{s+g K_{\theta_{\mathrm{i}}}}{s^{2}+a s+g K_{\theta_{\mathrm{i}}} a} .
$$


According to [15, Th. 7.1], we only have to show that $G(s)$ is strictly positive real to demonstrate that the interconnection is absolutely stable and that (8) is globally exponentially stable. We use [15, Lemma 6.1] to show that $G(s)$ is strictly positive real. The poles of $G(s)$ are given by $(-a \pm$ $\left.\sqrt{a^{2}-4 g K_{\theta_{\mathrm{i}}} a}\right) / 2$. For any $K_{\theta_{\mathrm{i}}}>0, \operatorname{Re}\left(\sqrt{a^{2}-4 g K_{\theta_{\mathrm{i}}} a}\right)<$ $a$; hence, $G(s)$ is Hurwitz. We find $\operatorname{Re}(G(j \omega))$ :

$$
\operatorname{Re}(G(j \omega))=\frac{\left(a-g K_{\theta_{\mathrm{i}}}\right) \omega^{2}+g^{2} K_{\theta_{\mathrm{i}}}^{2} a}{\left(g K_{\theta_{\mathrm{i}}} a-\omega^{2}\right)^{2}+a^{2} \omega^{2}} .
$$

If $K_{\theta_{\mathrm{i}}}<a / g$, then $a-g K_{\theta_{\mathrm{i}}}>0$, and hence $\operatorname{Re}(G(j \omega))>0$ for all $\omega \in \mathbb{R}$. We have that $G(\infty)=\lim _{s \rightarrow \infty} G(s)=0$. Hence, we must prove that $\lim _{\omega \rightarrow \infty} \omega^{2} \operatorname{Re}(G(j \omega))>0$. We have

$$
\omega^{2} \operatorname{Re}(G(j \omega))=\frac{\left(a-g K_{\theta_{\mathrm{i}}}\right) \omega^{4}+g^{2} K_{\theta_{\mathrm{i}}}^{2} a \omega^{2}}{\omega^{4}-2 g K_{\theta_{\mathrm{i}}} a \omega^{2}+g^{2} K_{\theta_{\mathrm{i}}}^{2} a^{2}+a^{2} \omega^{2}}
$$

Hence $\lim _{\omega \rightarrow \infty} \omega^{2} \operatorname{Re}(G(j \omega))=a-g K_{\theta_{\mathrm{i}}}>0$, which proves that $G(s)$ is strictly positive real, provided $K_{\theta_{\mathrm{i}}}<a / g$.

\section{B. Bank Angle}

We handle the bank angle in roughly the same way as the inclination angle. We define $\theta_{\mathrm{b}}=\cos (\Theta) \sin (\Phi)$ and introduce an estimate $\hat{\theta}_{\mathrm{b}}$ of $\theta_{\mathrm{b}}$. Typical bank and inclination angles are small enough that $\Phi \approx \theta_{\mathrm{b}}$, and $\hat{\theta}_{\mathrm{b}}$ is therefore considered an estimate of the bank angle. Using $\hat{\theta}_{\mathrm{b}}$ for compensation in the observer (3), we obtain

$$
\dot{\hat{v}}_{y}=a_{y}-\dot{\psi} \hat{v}_{x}-g \hat{\theta}_{\mathrm{b}}-K_{v_{y}}\left(a_{y}-\hat{a}_{y}\left(t, \hat{v}_{x}, \hat{v}_{y}\right)\right) .
$$

We again use the lateral acceleration as an indirect measurement of the lateral velocity, letting

$$
\dot{\hat{\theta}}_{\mathrm{b}}=K_{\theta_{\mathrm{b}}}\left(a_{y}-\hat{a}_{y}\left(t, \hat{v}_{x}, \hat{v}_{y}\right)\right),
$$

where $K_{\theta_{\mathrm{b}}}$ is a positive gain.

Ignoring the effect of the longitudinal-velocity error $\tilde{v}_{x}$, we write the error dynamics of the lateral-velocity and bankangle estimates as the interconnection of the LTI system

$$
\begin{aligned}
& \dot{\tilde{v}}_{y}=-K_{v_{y}} \delta_{\min } \tilde{v}_{y}-g \tilde{\theta}_{\mathrm{b}}+K_{v_{y}} u, \\
& \dot{\tilde{\theta}}_{\mathrm{b}}=K_{\theta_{\mathrm{b}}} \delta_{\min } \tilde{v}_{y}-K_{\theta_{\mathrm{b}}} u
\end{aligned}
$$

with the time-varying sector nonlinearity $u=-\left(\delta\left(t, \tilde{v}_{y}\right)-\right.$ $\left.\delta_{\text {min }}\right) \tilde{v}_{y}$.

Lemma 2: If the gain $K_{\theta_{\mathrm{i}}}$ is chosen such that $0<K_{\theta_{\mathrm{i}}}<$ $K_{v_{y}}^{2} \delta_{\min } / g$, then the origin of (11) is globally exponentially stable.

Proof (Outline): The function $\phi_{y}\left(t, \tilde{v}_{y}\right)=\left(\delta\left(t, \tilde{v}_{y}\right)-\right.$ $\left.\delta_{\min }\right) \tilde{v}_{y}$ belongs to the sector $[0, \infty]$, because $\phi_{y}\left(t, \tilde{v}_{y}\right) \tilde{v}_{y} \geq$ 0 . The proof proceeds as the proof of Lemma 1, with the transfer function

$$
G(s)=\frac{K_{v_{y}} s+g K_{\theta_{\mathrm{b}}}}{s^{2}+K_{v_{y}} \delta_{\min } s+g K_{\theta_{\mathrm{b}}} \delta_{\min }} .
$$

The resulting gain requirement is $K_{\theta_{\mathrm{b}}}<K_{v_{y}}^{2} \delta_{\min } / g$.

\section{Combined Analysis}

We have so far ignored the interconnections between the $\left(\tilde{v}_{x}, \tilde{\theta}_{\mathrm{i}}\right)$-subsystem and the $\left(\tilde{v}_{y}, \tilde{\theta}_{\mathrm{b}}\right)$-subsystem. With the help of Lemmas 1 and 2, we can now state a theorem for the combined system, taking the interconnections into account.

Theorem 1: There exists a constant $C>0$ such that if the gains $K_{\theta_{\mathrm{i}}}, K_{\theta_{\mathrm{b}}}$, and $K_{v_{x}}(t)$ are chosen such that $0<$ $K_{\theta_{\mathrm{i}}}<a / g, 0<K_{\theta_{\mathrm{b}}}<K_{v_{y}}^{2} \delta_{\min } / g$, and $K_{v_{x}, \min }>C$, then the origin of the error dynamics for the observer (6), (7), (9), (10) is globally exponentially stable.

Proof: From the proof of [15, Th. 7.1] there is a Lyapunov function $V_{1}=\frac{1}{2} x_{1}^{\top} P_{1} x_{1}$, where $x_{1}:=\left[\tilde{v}_{x}, \tilde{\theta}_{\mathrm{i}}\right]^{\top}$, such that, along the trajectories of (8),

$$
\begin{aligned}
\dot{V}_{1} & \leq-\varepsilon_{1} x_{1}^{\top} P_{1} x_{1}-\tilde{v}_{x} \phi_{x}\left(t, \tilde{v}_{x}\right) \\
& \leq-\varepsilon_{1} x_{1}^{\top} P_{1} x_{1}-\left(K_{v_{x}, \min }-a\right) \tilde{v}_{x}^{2},
\end{aligned}
$$

for some $\varepsilon_{1}>0$. There is a also a Lyapunov function $V_{2}=\frac{1}{2} x_{2}^{\top} P_{2} x_{2}$, where $x_{2}:=\left[\tilde{v}_{y}, \tilde{\theta}_{\mathrm{b}}\right]^{\top}$, such that, along the trajectories of (11), $\dot{V}_{2} \leq-\varepsilon_{2} x_{2}^{\top} P_{2} x_{2}$, for some $\varepsilon_{2}>0$.

If we include the effect of $\tilde{v}_{y}$ on the $x_{1}$-subsystem, we get an extra perturbation $\left[\dot{\psi} \tilde{v}_{y}, 0\right]^{\top}$ on the righthand side in (8). If we include the effect of $\tilde{v}_{x}$ on the $x_{2}$-subsystem, we get an extra perturbation $\left[-\dot{\psi} \tilde{v}_{x}+\right.$ $\left.K_{v_{y}} \kappa\left(t, \tilde{v}_{x}, \tilde{v}_{y}\right) \tilde{v}_{x},-K_{\theta_{\mathrm{b}}} \kappa\left(t, \tilde{v}_{x}, \tilde{v}_{y}\right) \tilde{v}_{x}\right]^{\top}$ on the right-hand side in (11), where $\kappa\left(t, \tilde{v}_{x}, \tilde{v}_{y}\right)$ comes from Assumption 2, by writing $\tilde{a}_{y}\left(t, \tilde{v}_{x}, \tilde{v}_{y}\right)=a_{y}-\hat{a}_{y}\left(t, v_{x}, \hat{v}_{y}\right)+\hat{a}_{y}\left(t, v_{x}, \hat{v}_{y}\right)-$ $\hat{a}_{y}\left(t, \hat{v}_{x}, \hat{v}_{y}\right)=-\delta\left(t, \tilde{v}_{y}\right) \tilde{v}_{y}+\kappa\left(t, \tilde{v}_{x}, \tilde{v}_{y}\right) \tilde{v}_{x}$. Using the Lyapunov function $V=V_{1}+c V_{2}$, where $c>0$ is yet to be determined, we have

$$
\begin{aligned}
& \dot{V} \leq-\varepsilon_{1} \lambda_{\min }\left(P_{1}\right)\left\|x_{1}\right\|^{2}+m_{\dot{\psi}}\left\|P_{1}\right\|\left\|x_{1}\right\|\left\|x_{2}\right\| \\
& +c m\left\|P_{2}\right\|\left\|x_{2}\right\|\left|\tilde{v}_{x}\right|-\left(K_{v_{x}, \min }-a\right) \tilde{v}_{x}^{2}-c \varepsilon_{2} \lambda_{\min }\left(P_{2}\right)\left\|x_{2}\right\|^{2},
\end{aligned}
$$

where $m_{\dot{\psi}}$ is a bound on $|\dot{\psi}|, m$ is a bound on $\|[-\dot{\psi}+$ $\left.K_{v_{y}} \kappa\left(t, \tilde{v}_{x}, \tilde{v}_{y}\right),-K_{\theta_{\mathrm{b}}} \kappa\left(t, \tilde{v}_{x}, \tilde{v}_{y}\right)\right] \|$, and $\lambda_{\min }\left(P_{i}\right), i=1,2$, denotes the minimum eigenvalue of $P_{i}$. We can split the right-hand side of the inequality by writing $\dot{V} \leq-W_{1}-W_{2}$, where

$$
W_{1}=\left[\begin{array}{ll}
\left\|x_{1}\right\| & \left\|x_{2}\right\|
\end{array}\right]\left[\begin{array}{cc}
\varepsilon_{1} \lambda_{\min }\left(P_{1}\right) & -\frac{m_{\dot{\psi}}}{2}\left\|P_{1}\right\| \\
-\frac{m_{\dot{\psi}}}{2}\left\|P_{1}\right\| & \frac{c \varepsilon_{2}}{2} \lambda_{\min }\left(P_{2}\right)
\end{array}\right]\left[\begin{array}{l}
\left\|x_{1}\right\| \\
\left\|x_{2}\right\|
\end{array}\right],
$$

and

$$
W_{2}=\left[\begin{array}{ll}
\left\|x_{2}\right\| & \left|\tilde{v}_{x}\right|
\end{array}\right]\left[\begin{array}{ll}
\frac{c \varepsilon_{2}}{2} \lambda_{\min }\left(P_{2}\right) & -\frac{1}{2} c m\left\|P_{2}\right\| \\
-\frac{1}{2} c m\left\|P_{2}\right\| & K_{v_{x}, \min }-a
\end{array}\right]\left[\begin{array}{c}
\left\|x_{2}\right\| \\
\left|\tilde{v}_{x}\right|
\end{array}\right] .
$$

The first-order principal minor of the $2 \times 2$ matrix in the $W_{1}$ expression is $\varepsilon_{1} \lambda_{\min }\left(P_{1}\right)>0$. The second-order principal minor is $\frac{1}{2} c \varepsilon_{1} \varepsilon_{2} \lambda_{\text {min }}\left(P_{1}\right) \lambda_{\min }\left(P_{2}\right)-$ $\frac{1}{4} m_{\dot{\psi}}^{2}\left\|P_{1}\right\|^{2}$, which is made positive by letting $c>$ $m_{\dot{\psi}}^{2}\left\|P_{1}\right\|^{2} /\left(2 \varepsilon_{1} \varepsilon_{2} \lambda_{\min }\left(P_{1}\right) \lambda_{\min }\left(P_{2}\right)\right)$. Hence, the matrix is positive definite. The first-order principal minor of the $2 \times 2$ matrix in the $W_{2}$ expression is $\frac{1}{2} c \varepsilon_{2} \lambda_{\min }\left(P_{2}\right)>0$. The second-order principal minor is $\frac{1}{2} c \varepsilon_{2} \lambda_{\min }\left(P_{2}\right)\left(K_{v_{x}, \min }-a\right)-$ $\frac{1}{4} c^{2} m^{2}\left\|P_{2}\right\|^{2}$, which is made positive by letting $K_{v_{x}, \min }>$ $C:=a+c m^{2}\left\|P_{2}\right\|^{2} /\left(2 \varepsilon_{2} \lambda_{\min }\left(P_{2}\right)\right)$ Hence, both matrices are positive definite, meaning that $\dot{V}$ is negative definite. 
Summing up the results of the stability analysis, global exponential stability of the observer error is guaranteed if the gains $K_{\theta_{\mathrm{i}}}, K_{\theta_{\mathrm{b}}}$ are chosen sufficiently low, and the minimum value $K_{v_{x} \text {, min }}$ of $K_{v_{x}}(t)$ is chosen sufficiently high. Even though there is no upper limit on $K_{v_{x}}(t)$, it is of obvious interest to limit this gain, to avoid direct propagation of $v_{x \text {, ref }}$ to the estimate $\hat{v}_{x}$.

\section{UNKNOWN ROAD-SURFACE CONDITIONS}

The observer (6), (7), (9), (10) depends on the construction of a lateral-acceleration estimate $\hat{a}_{y}\left(t, \hat{v}_{x}, \hat{v}_{y}\right)$ as a function of measured signals and velocity estimates. This approach assumes that there is no uncertainty in the friction model with respect to the road-surface conditions. In reality, however, the friction model is sensitive to changes in the road-surface conditions for large tire slips. In [11], the design from [10] is altered to take unknown road-surface conditions into account, by estimating a friction parameter $\theta$ along with the velocities.

The longitudinal-velocity and inclination-angle estimates do not depend on the friction model; hence, no changes are necessary to (6), (7). We focus on the lateral-velocity and friction-parameter estimation from [11], given by

$$
\begin{aligned}
\dot{\hat{v}}_{y}= & a_{y}-\dot{\psi} \hat{v}_{x} \\
& +K_{v_{y}} \Lambda\left(t, \hat{v}_{x}, \hat{v}_{y}\right) \xi\left(t, \hat{v}_{x}, \hat{v}_{y}\right)\left(a_{y}-\hat{a}_{y}\left(t, \hat{v}_{x}, \hat{v}_{y}, \hat{\theta}\right)\right) \\
\dot{\hat{\theta}}= & K_{\theta} \Lambda\left(t, \hat{v}_{x}, \hat{v}_{y}\right) \hat{a}_{y}^{*}\left(t, \hat{v}_{x}, \hat{v}_{y}\right)\left(a_{y}-\hat{a}_{y}\left(t, \hat{v}_{x}, \hat{v}_{y}, \hat{\theta}\right)\right) .
\end{aligned}
$$

Here $\hat{a}_{y}$ has been redefined as $\hat{a}_{y}\left(t, \hat{v}_{x}, \hat{v}_{y}, \hat{\theta}\right)=$ $\hat{\theta} \hat{a}_{y}^{*}\left(t, \hat{v}_{x}, \hat{v}_{y}\right)$, where $\hat{a}_{y}^{*}\left(t, \hat{v}_{x}, \hat{v}_{y}\right)$ is the lateral-acceleration estimate from the friction model, obtained using a nominal parameterization corresponding to a high-friction surface. The estimated friction parameter $\hat{\theta}$ scales the output of the friction model to adapt to different road-surface conditions. The value $\xi\left(t, \hat{v}_{x}, \hat{v}_{y}\right)$ is the approximated slope of the line between $\left(\hat{v}_{y}, \hat{a}_{y}^{*}\left(t, \hat{v}_{x}, \hat{v}_{y}\right)\right)$ and $\left(v_{y}, \hat{a}_{y}^{*}\left(t, \hat{v}_{x}, v_{y}\right)\right)$, which is negative according to Assumption 1. The function $\Lambda\left(t, \hat{v}_{x}, \hat{v}_{y}\right)$ is a freely chosen, strictly positive scaling function, which we define as $\Lambda\left(t, \hat{v}_{x}, \hat{v}_{y}\right)=\left(\xi^{2}\left(t, \hat{v}_{x}, \hat{v}_{y}\right)+\right.$ $\left.\hat{a}_{y}^{*}\left(t, \hat{v}_{x}, \hat{v}_{y}\right)\right)^{-1 / 2}$ to give the observer good numerical properties. Under the assumptions in [11], the estimation error for (12) is uniformly globally asymptotically stable and locally exponentially stable. For details, we refer to [11].

A central assumption in [11] concerns the lateral excitation of the vehicle. In order to simultaneously estimate the lateral velocity and the friction parameter, there needs to be variation in the vehicle's lateral movement. During stationary maneuvers, such as straight driving, we cannot estimate the friction parameter along with the lateral velocity, and the friction estimation therefore has to be turned off.

\section{A. When to Estimate Friction}

Because lateral excitation is needed for the friction estimation, and because estimation of the friction parameter is unnecessary during normal driving with small tire slips, we leave the friction estimation off most of the time, and only turn it on in certain cases. When to turn the friction estimation on is determined in a manner similar to that of an ESC system and alternative approaches found in literature (for example [2], [16]). Using a linear reference model for the yaw rate, we determine when the car becomes over- or understeered. We also estimate $\dot{v}_{y}$, according to (1b), by $a_{y}-\dot{\psi} \hat{v}_{x}$, highpass-filtered with a 10 -s time constant. When $\dot{v}_{y}$ is high, it indicates a fast-changing sideslip angle, which in turn indicates a high level of excitation and that some of the tires might be in the nonlinear region. When the estimate of $\dot{v}_{y}$ is high, and the reference yaw rate is above a threshold value, we therefore turn the friction estimation on. We also turn the friction estimation on when the car is oversteered; and when the vehicle's ESC system is active, but not due to stationary understeer. A small delay in turning friction estimation off reduces chattering in the friction estimation condition.

\section{B. Combining Friction Estimation with Bank-Angle Estima- tion}

We can modify (12a) to take the bank angle into account, by redefining

$$
\begin{aligned}
\dot{\hat{v}}_{y}= & a_{y}-\dot{\psi} \hat{v}_{x}-g \hat{\theta}_{\mathrm{i}} \\
& +K_{v_{y}} \Lambda\left(t, \hat{v}_{x}, \hat{v}_{y}\right) \xi\left(t, \hat{v}_{x}, \hat{v}_{y}\right)\left(a_{y}-\hat{a}_{y}\left(t, \hat{v}_{x}, \hat{v}_{y}, \hat{\theta}\right)\right) .
\end{aligned}
$$

Because the lateral-velocity estimation in (13) is different from (9), we also alter the bank-angle estimation to make it fit within the absolute-stability framework:

$\dot{\hat{\theta}}_{\mathrm{b}}=-K_{\theta_{\mathrm{b}}} \Lambda\left(t, \hat{v}_{x}, \hat{v}_{y}\right) \xi\left(t, \hat{v}_{x}, \hat{v}_{y}\right)\left(a_{y}-\hat{a}_{y}\left(t, \hat{v}_{x}, \hat{v}_{y}, \hat{\theta}\right)\right)$.

In the previous Lyapunov analysis, we have used tunable gains to dominate cross-terms that appear in the time derivative of the Lyapunov function. A Lyapunov function is available from [11] to prove exponential stability of the lateral-velocity and friction-parameter error, within an arbitrarily large region of attraction around the origin. This Lyapunov function can be combined with the Lyapunov function for the bank-angle estimation error. However, the stability margin from [11] depends on the level of excitation, which cannot be changed by increasing the gains, and the conditions for asymptotic stability are therefore difficult or impossible to verify. We therefore cannot find a particular set of observer gains to guarantee asymptotic stability when friction estimation is combined with bank-angle estimation.

From a practical point of view, it is often difficult to distinguish the effect of low friction and a nonzero bank angle in experimental data, something that is also noted in [17]. This observation suggests that the difficulties we encounter in analyzing the combined approach reflects a genuine observability problem. How to combine friction estimation and bank-angle estimation is therefore a matter of practical consideration.

The simplest strategy would be to turn the bank-angle estimation off whenever friction estimation is turned on. With this strategy, however, it is possible to provoke problems when driving on slippery surfaces in hilly terrains, where combinations of large vehicle sideslip angles and large bank angles may occur. For this reason, we leave the bank-angle 
estimation on when estimating friction, albeit with the gain set lower whenever one of three conditions hold. The first condition is that $\left|a_{y}-\hat{a}_{y}\left(t, \hat{v}_{x}, \hat{v}_{y}, \hat{\theta}\right)\right|>c_{1}$ for some $c_{1}>0$. The second condition is that $\left|\ddot{\psi}-\hat{\ddot{\psi}}\left(t, \hat{v}_{x}, \hat{v}_{y}, \hat{\theta}\right)\right|>c_{2}$ for some $c_{2}>0$, where $\ddot{\psi}$ is the yaw acceleration found by numerical differentiation of the yaw rate, and $\hat{\ddot{\psi}}\left(t, \hat{v}_{x}, \hat{v}_{y}, \hat{\theta}\right)$ is calculated using the friction model in the same way as $\hat{a}_{y}\left(t, \hat{v}_{x}, \hat{v}_{y}, \hat{\theta}\right)$, with the forces scaled by $\hat{\theta}$. The third condition is that $\operatorname{sign}\left(\dot{\psi}_{\text {ref }}\right)=\operatorname{sign}(\dot{\psi})$ and $\left(\left|\dot{\psi}_{\text {ref }}\right|-|\dot{\psi}|\right) \hat{v}_{x}>$ $c_{3}$ for some $c_{3}>0$, where $\dot{\psi}_{\text {ref }}$ is the reference yaw rate used in the friction estimation conditions described in Section VA. From experimental data, these conditions are found to reduce the negative interference of the bank-angle estimation on low-friction surfaces. We emphasize, however, that these conditions are purely practical and that alternative conditions may be equally good or better.

\section{Final Observer}

The final observer that we end up with has two modes, without friction estimation and with friction estimation. Without friction estimation we use equations (6), (7), (9), (10), where $\hat{a}_{y}\left(t, \hat{v}_{x}, \hat{v}_{y}\right)$ is replaced by the frictionparameter dependent $\hat{a}_{y}\left(t, \hat{v}_{x}, \hat{v}_{y}, \hat{\theta}\right)$, and the friction parameter is attracted to a high default value: $\dot{\hat{\theta}}=K_{\mathrm{e}}\left(\theta^{*}-\hat{\theta}\right)$. With friction estimation we use (6), (7), (13), (12b), (14).

In both modes, $K_{\theta_{\mathrm{b}}}$ is reduced according to the practical conditions described above. Based on experimental results, we also choose to set the gain $K_{\theta_{\mathrm{i}}}$ for the inclination-angle estimation lower for the second mode than for the first.

\section{SOME REMARKS ABOUt RoAd-TIRE FRICTION}

Throughout this paper, we assume a strictly monotonic relationship between the lateral acceleration and the lateral velocity. For large tire slip angles, however, the road-tire friction forces saturate completely, and may decrease slightly. In certain extreme situations with large tire slips for all tires, the monotonicity assumption may therefore fail to hold. Reflecting this possibility, the stability results in [10] are stated as regional rather than global. We have chosen to simplify the analysis by operating in the global domain. In the friction model used in the experiments, the friction forces saturate for large tire slip angles, but do not decrease. This helps ensure stability of the observer equations.

\section{IMPLEMENTATION AND EXPERIMENTAL RESULTS}

The observer is discretized using the forward-Euler method with a 10-ms sample time. The measurements are taken from production-type sensors, and are filtered with a $15-\mathrm{Hz}$ lowpass filter, without any correction for sensor bias. Some notable implementation details are the following:

- The wheel loads are calculated using the acceleration measurements, as in [14, Ch. 7.4].

- Tire relaxation dynamics are approximated in a manner similar to [13, Ch. 1], by filtering the output of the friction model with a transfer function $1 /(T s+1)$, where $T=T_{e}+l_{e} / \hat{v}_{x}$.
- The front-axle steering angles are calculated using the measured steering wheel angle and a lookup table based on a steering transmission curve. The effect of caster is included by making the steering angles dependent on the estimated lateral road-tire friction forces for the front axle, according to [13, Ch. 1].

- The effect of vehicle roll on the lateral-acceleration measurement is compensated by multiplying the measured lateral acceleration with $1 /\left(1+p_{g} g\right)$, where $p_{g}$ is the roll-angle gradient.

- As in [11], the range of possible friction parameters is limited. The estimated friction parameter must belong to $[0.05,1.1]$ and, in addition, we ensure that $1.2 g \hat{\theta} \geq$ $\left(a_{x}^{2}+a_{y}^{2}\right)^{1 / 2}$. We do so because $1.2 \hat{\theta} g$ is the approximate maximum acceleration achievable from the friction model when multiplied by the friction parameter, and this maximum should never be smaller than the actual acceleration of the vehicle.

The first experiment is a circle maneuver with a 40-m radius, carried out on dry asphalt. The longitudinal velocity is slowly increased from about $10 \mathrm{~km} / \mathrm{h}$ to about $65 \mathrm{~km} / \mathrm{h}$, when the circle can no longer be maintained because of severe understeer. The area on which the circle maneuver takes place has a constant slope of $0.5^{\circ}$; hence, both the inclination angle and the bank angle oscillate between $\pm 0.5^{\circ}$ as the maneuver takes place. The results are shown in Fig. 2. Until approximately $88 \mathrm{~s}$ into the test, the bank angle follows the reference well, albeit with a phase lag. The phase lag can be made smaller by increasing the gain, but this is not desirable for the sideslip angle estimate. After $88 \mathrm{~s}$, the bank-angle estimate reacts slowly to changes, because the gain is then reduced according to the practical conditions in Section V-B. There is also a small offset in the bank angle, attributable to offsets in the measurements of the steering wheel angle, lateral acceleration, and yaw rate. For the inclination angle there is a significant offset, caused by an offset in the longitudinal-acceleration sensor. Nevertheless, the estimated sideslip angle is accurate.

The second experiment consists of driving downhill from a mountain top on snow-covered roads. In this experiment, we have no reference for the inclination and bank angles; hence we have to evaluate the estimates based on plausibility and on the quality of the estimated sideslip angle. The results are shown in Fig. 3. Qualitatively, the estimates match well with the terrain, indicating a varying but steep descent, and significant road bank angles. The resulting sideslip angle estimate is accurate.

It is evident from Fig. 2 that offsets found in productiontype sensors have a significant impact on the achievable accuracy of the inclination- and bank-angle estimation. Correction for inertial sensor offsets is a fundamental, challenging problem that lies outside the scope of this paper.

\section{CONCLUding REMARKS}

The primary reason for estimating the road inclination and bank angle is to improve estimation of the sideslip angle on slanted road surfaces. In this respect, experimental results are 


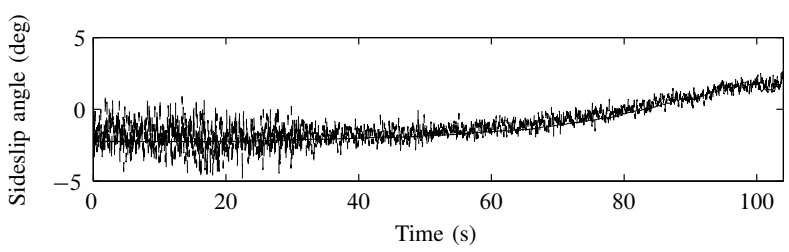

(a) Real (dashed) and estimated (solid) sideslip angle

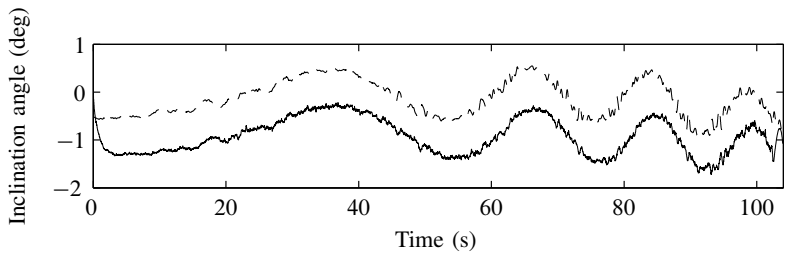

(b) Real (dashed) and estimated (solid) inclination angle

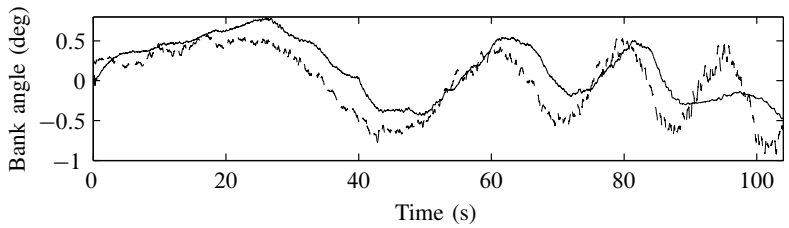

(c) Real (dashed) and estimated (solid) bank angle

Fig. 2. Experimental results for circle maneuver

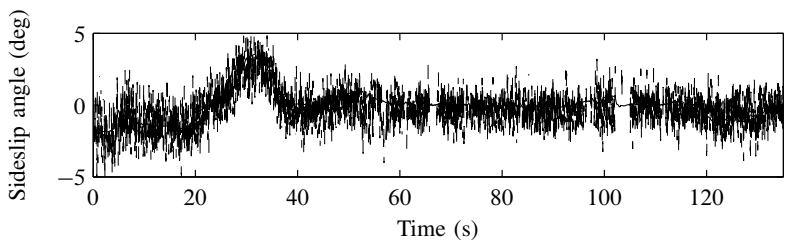

(a) Real (dashed) and estimated (solid) sideslip angle

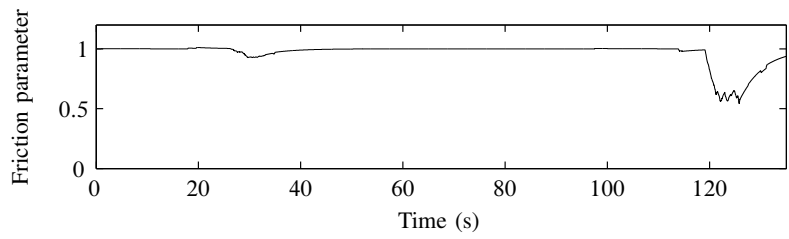

(b) Estimated friction parameter

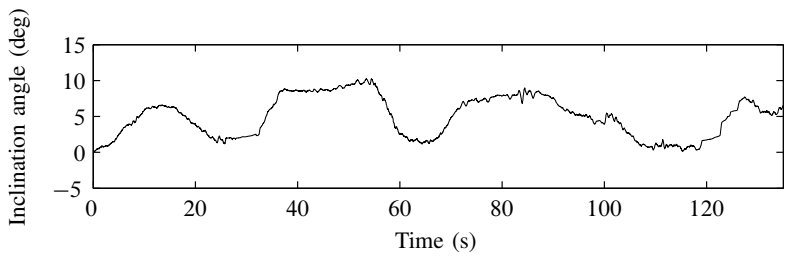

(c) Estimated inclination angle

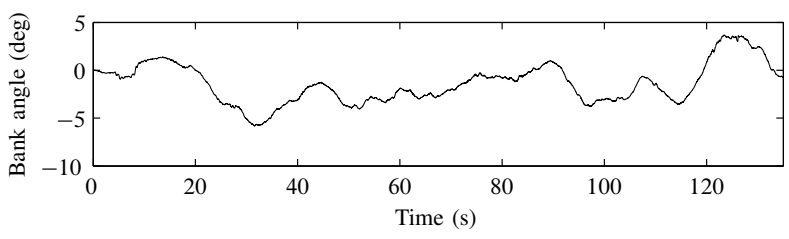

(d) Estimated bank angle

Fig. 3. Experimental results for mountain driving mostly good; in most situations, the estimates of the sideslip angle are of high quality. Problems persist, however, in some situations on low-friction surfaces. The problems can largely be attributed to the difficulty of distinguishing a nonzero bank angle from low friction. The method of combining friction estimation and bank-angle estimation is a weakness of the present design, which limits the achievable performance. The ad-hoc approach of selectively reducing the bank angle gain based on various criteria is likely to need revision and improvement for the observer to reach production quality. Preliminary results indicate that eventual availability of sixdegree-of-freedom inertial sensors in production vehicles is likely to help, by making estimation of the bank angle less dependent on the friction model.

With respect to providing accurate estimates of the inclination and bank angle, the approach gives plausible estimates in most normal driving situation, but sensor offsets and other slowly-varying errors propagate to the inclination- and bankangle estimates. In extreme situations with large tire slip angles, the estimates often become temporarily implausible, due primarily to inaccuracies in the friction model.

\section{REFERENCES}

[1] A. Suissa, Z. Zomotor, and F. Böttiger, "Method for determining variables characterizing vehicle handling," US Patent 5,557,520, 1994 , filed Jul. 29, 1994; issued Sep. 17, 1996.

[2] Y. Fukada, "Slip-angle estimation for vehicle stability control," Vehicle Syst. Dyn., vol. 32, no. 4, pp. 375-388, 1999.

[3] W. Klier, A. Reim, and D. Stapel, "Robust estimation of vehicle sideslip angle - an approach w/o vehicle and tire models," in Proc. SAE World Congress, Detroit, MI, 2008, paper no. 2008-01-0582.

[4] H. E. Tseng, "Dynamic estimation of road bank angle," Vehicle Syst. Dyn., vol. 36, no. 4, pp. 307-328, 2001.

[5] C. Sentouh, Y. Sebsadji, S. Mammar, and S. Glaser, "Road bank angle and faults estimation using unknown input proportional-integral observer," in Proc. Eur. Contr. Conf., Kos, Greece, 2007, pp. 51315138

[6] Y. Sebsadji, S. Glaser, S. Mammar, and M. Netto, "Vehicle roll and road bank angles estimation," in Proc. 17th IFAC World Congress, Seoul, South Korea, 2008, pp. 7091-7097.

[7] D. M. Bevly, "Global Positioning System (GPS): A low-cost velocity sensor for correcting inertial sensor errors on ground vehicles," J. Dyn. Syst. Meas. Contr., vol. 126, no. 2, pp. 255-264, 2004.

[8] J. Ryu and J. C. Gerdes, "Estimation of vehicle roll and road bank angle," in Proc. Am. Contr. Conf., Boston, MA, 2004, pp. 2110-2115.

[9] R. G. Brown and P. Y. C. Hwang, Introduction to Random Signals and Applied Kalman Filtering, 3rd ed. Wiley, 1997.

[10] L. Imsland, T. A. Johansen, T. I. Fossen, H. F. Grip, J. C. Kalkkuhl, and A. Suissa, "Vehicle velocity estimation using nonlinear observers," Automatica, vol. 42, no. 12, pp. 2091-2103, 2006.

[11] H. F. Grip, L. Imsland, T. A. Johansen, T. I. Fossen, J. C. Kalkkuhl, and A. Suissa, "Nonlinear vehicle side-slip estimation with friction adaptation," Automatica, vol. 44, no. 3, pp. 611-622, 2008.

[12] L. Imsland, T. A. Johansen, H. F. Grip, and T. I. Fossen, "On nonlinear unknown input observers - applied to lateral vehicle velocity estimation on banked roads," Int. J. Contr., vol. 80, no. 11, pp. 17291740, 2007.

[13] H. B. Pacejka, Tire and Vehicle Dynamics, 2nd ed. SAE International, 2005

[14] U. Kiencke and L. Nielsen, Automotive Control Systems. Springer 2000.

[15] H. K. Khalil, Nonlinear Systems, 3rd ed. Prentice Hall, 2002.

[16] A. Hac and M. D. Simpson, "Estimation of vehicle side slip angle and yaw rate," in Proc. SAE World Congress, Detroit, MI, 2000, paper no. 2000-01-0696.

[17] A. T. van Zanten, "Bosch ESP systems: 5 years of experience," in Proc. Automot. Dyn. Stabil. Conf., Troy, MI, 2000, paper no. 200001-1633. 